University of Northern lowa

UNI ScholarWorks

\title{
High-Energy Kink Observed in the Electron Dispersion of High- Temperature Cuprate Superconductors
}

\author{
T. Valla \\ Brookhaven National Laboratory \\ T. E. Kidd \\ University of Northern lowa \\ See next page for additional authors
}

Let us know how access to this document benefits you

Copyright @2007 T. Valla, T.E. Kidd, W.-G. Yin, G.D. Gu, P.D. Johnson, Z.-H. Pan, and A.V. Fedorov. The copyright holder has granted permission for posting.

Follow this and additional works at: https://scholarworks.uni.edu/phy_facpub

Part of the Physics Commons

\section{Recommended Citation}

Valla, T.; Kidd, T. E.; Yin, W.-G.; Gu, G. D.; Pan, Z.-H.; and Fedorov, A. V., "High-Energy Kink Observed in the Electron Dispersion of High-Temperature Cuprate Superconductors" (2007). Faculty Publications. 12. https://scholarworks.uni.edu/phy_facpub/12

This Article is brought to you for free and open access by the Faculty Work at UNI ScholarWorks. It has been accepted for inclusion in Faculty Publications by an authorized administrator of UNI ScholarWorks. For more information, please contact scholarworks@uni.edu. 
Authors

T. Valla, T. E. Kidd, W.-G. Yin, G. D. Gu, Z.-H. Pan, and A. V. Fedorov 


\title{
High-Energy Kink Observed in the Electron Dispersion of High-Temperature Cuprate Superconductors
}

\author{
T. Valla, ${ }^{1, *}$ T. E. Kidd, ${ }^{1,2}$ W.-G. Yin, ${ }^{1}$ G. D. Gu, ${ }^{1}$ P. D. Johnson, ${ }^{1}$ Z.-H. Pan, ${ }^{3}$ and A. V. Fedorov ${ }^{3}$ \\ ${ }^{1}$ Condensed Matter Physics and Materials Science Department, Brookhaven National Laboratory, Upton, New York 11973, USA \\ ${ }^{2}$ Physics Department, University of Northern Iowa, Cedar Falls, Iowa 50614-0150, USA \\ ${ }^{3}$ Advanced Light Source, Lawrence Berkeley National Laboratory, Berkeley, California 94720, USA
}

(Received 17 October 2006; published 18 April 2007)

Photoemission studies show the presence of a high-energy anomaly in the observed band dispersion for two families of cuprate superconductors, $\mathrm{Bi}_{2} \mathrm{Sr}_{2} \mathrm{CaCu}_{2} \mathrm{O}_{8+\delta}$ and $\mathrm{La}_{2-x} \mathrm{Ba}_{x} \mathrm{CuO}_{4}$. The anomaly, which occurs at a binding energy of approximately $340 \mathrm{meV}$, is found to be anisotropic and relatively weakly doping dependent. Scattering from short range or nearest neighbor spin excitations is found to supply an adequate description of the observed phenomena.

In conventional metals, electron-phonon coupling, or the phonon-mediated interaction between electrons, has long been known to be the pairing interaction responsible for the superconductivity. The strength of this interaction essentially determines the superconducting transition temperature $T_{c}$. One manifestation of electron-phonon coupling is a mass renormalization of the electronic dispersion at the energy scale associated with the phonons. This renormalization is directly observable in photoemission experiments [1]. In contrast, there remains little consensus on the pairing mechanism in cuprate high-temperature superconductors (HTSC) . The recent observation of similar renormalization effects in cuprates has raised the hope that the mechanism of high-temperature superconductivity may finally be resolved [2-9]. The focus has been on the lowenergy renormalization and associated "kink" in the dispersion at around $50 \mathrm{meV}$. However, at that energy scale, there are multiple candidates including phonon branches, structure in the spin-fluctuation spectrum, and the superconducting gap itself making the unique identification of the excitation responsible for the kink difficult. Here we show that the low-energy renormalization at $\sim 50 \mathrm{meV}$ is only a small component of the total renormalization, the majority of which occurs at nearly an order of magnitude higher energy $(\sim 340 \mathrm{meV})$. Two recent studies have also reported similar behavior [10,11]. The high-energy kink poses new challenges for the physics of the cuprates: what, if any, is its role in superconductivity and what is its relationship to the low-energy kink? In this Letter we explore these issues and show that interaction with the short wavelength nearest neighbor spin excitations provides a good description of the phenomena observed at higher energies.

The experiments reported here were carried out on a Scienta SES-2002 electron spectrometer at beam line U13UB of the National Synchrotron Light Source and on a Scienta SES-100 spectrometer at beam line 12.0.1 of the Advanced Light Source. The combined instrumental energy resolution was $\sim 20 \mathrm{meV}(\sim 35 \mathrm{meV})$ at $21(110) \mathrm{eV}$ photon energy used at U13UB (12.0.1), and the momentum resolution was $\pm 0.004( \pm 0.015) \AA^{-1}$, correspondingly. Samples, grown by the traveling solvent floating zone method, were mounted on a liquid $\mathrm{He}$ cryostat and cleaved in situ in the UHV chamber with a base pressure $2 \times$ $10^{-9} \mathrm{~Pa}$. The temperature was measured using a calibrated silicon sensor mounted near the sample. The photoemission spectra were analyzed using momentum distribution curves (MDC).

Figure 1 shows wide energy range angle-resolved photoemission spectra for two different families of cuprate HTSCs at different doping levels including optimally doped $\left(T_{c}=91 \mathrm{~K}\right)$ and highly underdoped $\left(T_{c}=5 \mathrm{~K}\right)$ $\mathrm{Bi}_{2} \mathrm{Sr}_{2} \mathrm{CaCu}_{2} \mathrm{O}_{8+\delta}$ (BSCCO) and $\mathrm{La}_{2-x} \mathrm{Ba}_{x} \mathrm{CuO}_{4}$ $(\mathrm{LBCO})$ at $x=0.095\left(T_{c}=32 \mathrm{~K}\right)$ and $x=1 / 8\left(T_{c}=\right.$ $2.5 \mathrm{~K})$. Spectra were taken from several different lines in $k$ space at $\sim 10 \mathrm{~K}$. This temperature corresponds to the normal state for underdoped BSCCO and $x=1 / 8 \mathrm{LBCO}$ and the superconducting state for optimally doped BSCCO and LBCO at $x=0.095$. In the latter cases, the familiar low-energy kink can easily be seen at approximately $50 \mathrm{meV}$. However, another, far more pronounced, kink can be seen in all the samples at much higher binding energies. Measurements of the intensity as a function of momentum or MDC [1,2] show relatively well-defined peaks down to $0.8 \mathrm{eV}$ that can be fitted with Lorentzian line shapes. The MDC derived dispersions clearly show kinks at high energies in the range around $300-400 \mathrm{meV}$, while at even higher energies $(\sim 0.7 \mathrm{eV})$ they tend to recover the bare tight-binding (TB) dispersions [12]. In the energy range between these two limits, the MDC peaks show very steep, and in the BSCCO case, virtually "vertical dispersion" near $(\pi / 4, \pi / 4)$. The vertical dispersion also appears in the LBCO system in the majority of momentum space, except near the nodal line. In the latter region, the rate of dispersion increases at high energies, but never becomes vertical. However, we note that the wave vector associated with the vertical dispersion is not common to all the cuprates - rather, it depends on doping and on details of the relevant "band structure." 

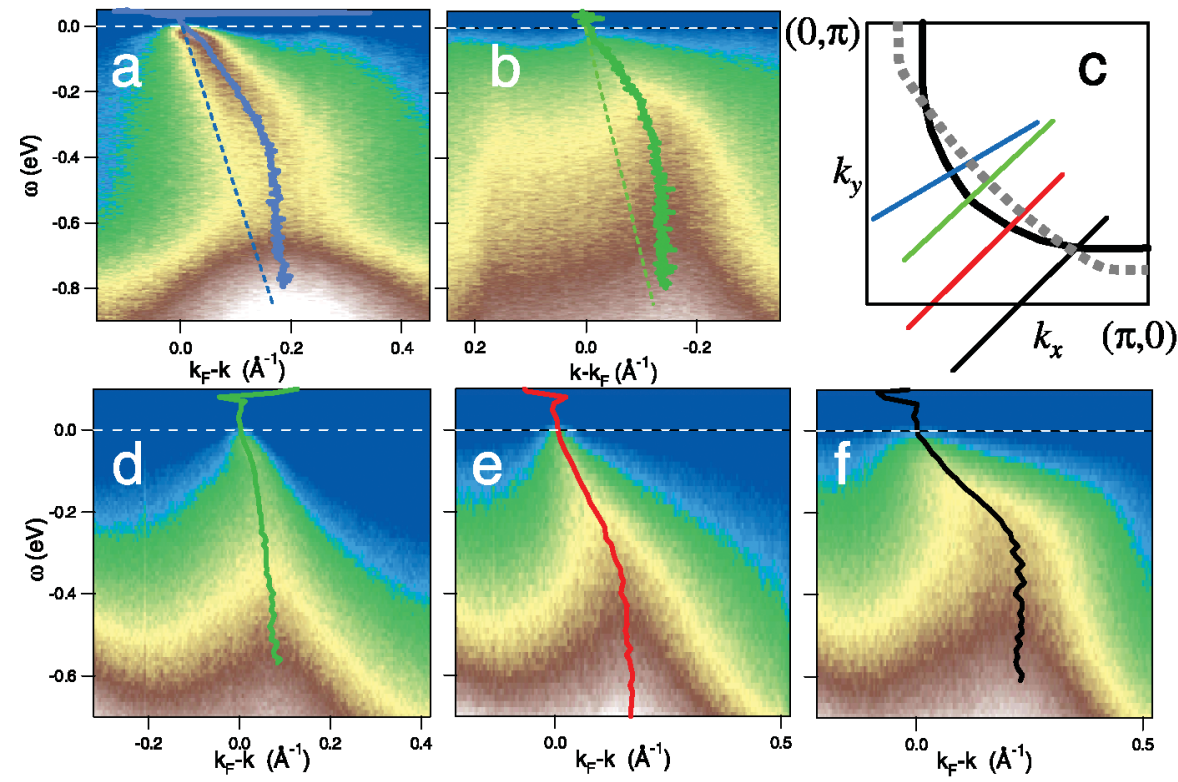

FIG. 1 (color). Photoemission spectra recorded from (a) optimally doped $\mathrm{BSCCO}$ and $(\mathrm{b})$ very underdoped $\left(T_{c} \sim\right.$ $5 \mathrm{~K}) \mathrm{BSCCO}$. (c) Brillouin zone with the Fermi surfaces for BSCCO (solid line) and LBCO (dashed line). Straight lines represent the momentum lines probed in the spectra with correspondingly colored dispersion. (d) Nodal LBCO spectrum for $x=0.095$. (e),(f) LBCO spectra at $x=0.125$ for two different momentum lines as indicated in (c). Dashed lines in the spectra represent bare tight-binding dispersions while solid lines represent MDC derived dispersions.
This observation is illustrated in Fig. 2 where we show in (a) and (b) the spectral intensity at the Fermi level for two different doping levels of LBCO system. The $x=$ 0.165 sample is superconducting with $T_{c}=24 \mathrm{~K}$. In (c) and (d) we show the corresponding contours at $\omega=$ $-0.4 \mathrm{eV}$. The latter contours represent the momentum positions corresponding to the vertical dispersion. The contours stay nearly fixed in the energy range between $300-450 \mathrm{meV}$ (except in the vicinity of the nodal line) but differ from one doping level to another and are all far from the $(\pi / 4, \pi / 4)$ contour, characteristic of BSCCO samples. In Fig. 2(e) we show the momentum $k_{0}$ characterizing the contours for the measured LBCO samples and compare it with corresponding $k_{0}$ magnitudes from a similar study of BSCCO [10]. The authors in the latter study suggest that the $(\pi / 4, \pi / 4)$ contour may imply some form of antiferromagnetic ordering with corresponding fourfolding of the Brillouin zone. We also show in the figure the results of a study of the undoped parent compound $\mathrm{Ca}_{2} \mathrm{CuO}_{2} \mathrm{Cl}_{2}(\mathrm{CCOC})$ where the high-energy kink has been observed at even slightly higher energy $\sim 450 \mathrm{meV}$ measured from the top of valence band [13]. This latter system is an antiferromagnetic correlated insulator with welldefined magnetic excitations or magnons extending up to several hundred meV.

The shift toward $(\pi / 2, \pi / 2)$ or much larger $k_{0}$ vectors in the 214 [LBCO and $\mathrm{La}_{2-x} \mathrm{Sr}_{x} \mathrm{CuO}_{4}$ (LSCO)] samples relative to BSCCO seems to be a simple consequence of the different bare dispersions and Fermi surface shapes for these two families of cuprates. Because of a smaller next nearest neighbor hopping $t^{\prime}$ in the 214 systems the constant energy contours at low energies are less curved and tend to be closer to the $(\pi / 2, \pi / 2)$ point than in the BSCCO family. Therefore, the vertical dispersion is pushed closer to $(\pi / 2, \pi / 2)$ in the 214 systems. This suggests that the observed high-energy kink is not an "ordering effect," but that it rather represents a true renormalization and opens the possibility of extracting the complete self-energy.

The real and imaginary components of the self-energy are derived in the usual manner from the widths $\Delta k$ and peak positions $k_{m}$ of the MDC peaks using the expressions

$$
\begin{aligned}
k_{m} & =k_{F}+[\omega-\operatorname{Re} \Sigma(\omega)] / v_{0} \\
\text { and } \quad \Delta k & =2 \operatorname{Im} \Sigma(\omega) / v_{0} .
\end{aligned}
$$

The MDCs are Lorentzians if the bare dispersion is linear, $\varepsilon_{k}=v_{0}\left(k-k_{F}\right)$, and $\Sigma$ does not vary strongly with momentum, an approximation that works well in the cuprates. Here $\operatorname{Re} \Sigma(\omega)$ and $\operatorname{Im} \Sigma(\omega)$ represent the real and imaginary components of the self-energy at a binding energy $\omega$ and $v_{0}$ represents the bare velocity. The results
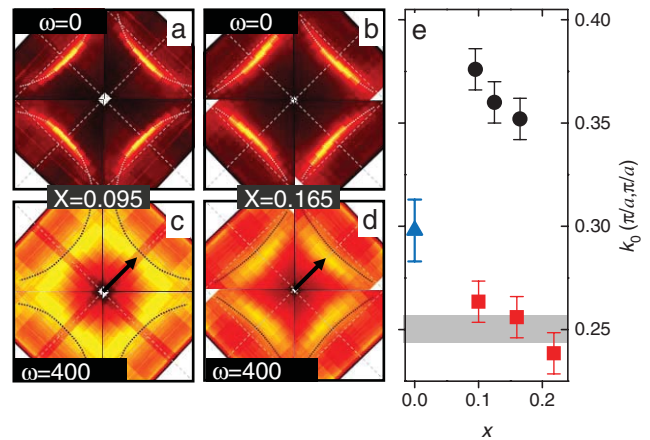

FIG. 2 (color). Characteristic momenta measured for the Fermi surfaces of LBCO at (a) $x=0.095$ and (b) $x=0.165$, respectively. (c),(d) Show corresponding intensity contours at $\omega=-0.4 \mathrm{eV}$. The arrows indicate the wave vector $k_{0}$ of a peak in MDC at $\omega=-0.4 \mathrm{eV}$ measured from the zone center along the zone diagonal. (e) Magnitude of $k_{0}$ where vertical dispersion occurs for LBCO from this study (circles), BSCCO from Ref. [10] (squares), and for CCOC from Ref. [13] (triangle). Shaded region marks $(\pi / 4, \pi / 4)$ wave vector, suggested in [10] to be an "ordering" vector. 
are shown in Fig. 3. Figures 3(a) and 3(b) show the widerange $\operatorname{Re} \Sigma(\omega)$ for different doping levels of BSCCO and LBCO, respectively. For the noninteracting dispersions indicated in Fig. 1, we have used linear approximations based on the TB derived Fermi velocities for each probed line [12]. As the TB dispersions depart from linear approximations at higher binding energies, decreasing velocities would reduce the self-energies [14]. However, this effect is relatively small within the range of $|\omega| \sim 0.5 \mathrm{eV}$ and does not affect the global picture presented here. Note that Eq. (1) is already a crude approximation as it assumes $k$-independent self-energies. The resulting self-energies shown in Fig. 3 are almost 1 order of magnitude larger than those previously reported in studies focused on the low-energy renormalization and have dominant structures at $\sim 340 \mathrm{meV}$. We note that the measured self-energies show several interesting characteristics: the high-energy kink in the LBCO system strengthens away from the nodal line. A similar result has recently been reported for the BSCCO system [11]. However, in contrast to the latter system where only the amplitude varies, while the overall shape of $\operatorname{Re} \Sigma$ remains nearly $k$ independent, in the LBCO system the peak position and the shape of $\operatorname{Re} \Sigma$ depend on $k$. For a given momentum line, the high-energy renormalization is stronger in BSCCO than in LBCO and in both systems only marginally dependent on doping.

The presence of a kink at these high energies immediately raises the question, What type of excitation spectrum is required to produce such a renormalization effect? We note that the phonon distribution in these materials is limited to $\sim 80 \mathrm{meV}$. Furthermore, the superconducting gap and the so-called "pseudogap" are doping dependent and usually always less than $50 \mathrm{meV}$ in $\mathrm{Bi}_{2} \mathrm{Sr}_{2} \mathrm{CaCu}_{2} \mathrm{O}_{8+\delta}$,

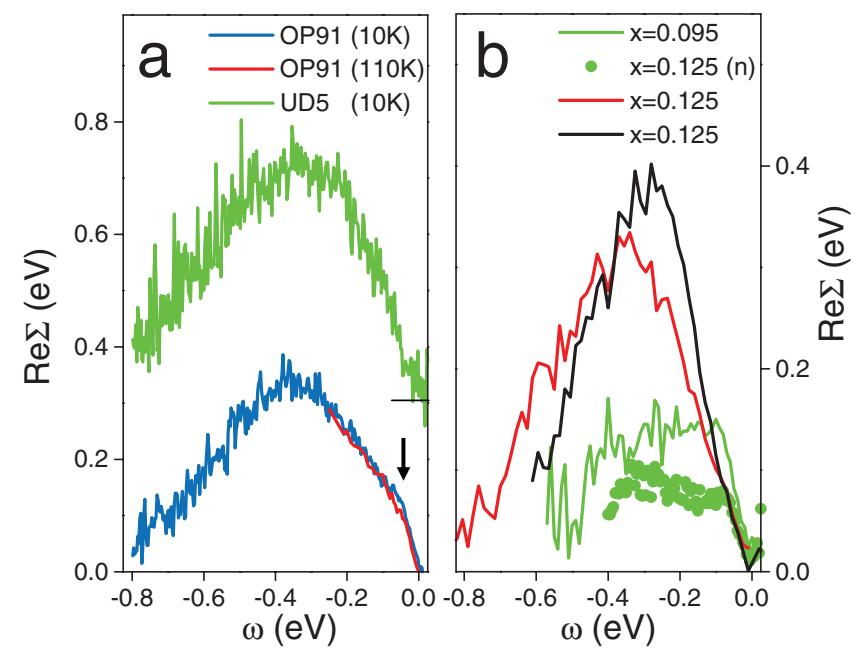

FIG. 3 (color). $\operatorname{Re} \Sigma$ for samples and lines indicated in Fig. 1. (a) BSCCO and (b) LBCO. Green line in (a) is shifted up by $0.3 \mathrm{eV}$. The arrow indicates the low-energy region where changes occur between the normal and superconducting state. Green circles in (b) represent the nodal $\operatorname{Re} \Sigma$ for $x=0.125 \mathrm{LBCO}$ (not shown in Fig. 1). Colors correspond to those used in Fig. 1. and even smaller in LBCO [15]. Therefore, both phonons and (pseudo)gaps can clearly be ruled out as the origin of the large renormalization observed at high energies. The only excitations that are available that extend to these energies are spin fluctuations, or magnons, in the undoped material [16-19].

In Fig. 4(a) we present a model distribution of "bosonic excitations" that would allow the reproduction of the selfenergy characteristics presented in Fig. 3 for the optimally doped BSCCO in the superconducting state. Essentially, three features are required: a relatively narrow peak at low energy $\sim 50-70 \mathrm{meV}$, a rather broad peak centered at $\sim 340 \mathrm{meV}$, and a continuum in the region in between. We note in passing that the narrow peak at low energies accounts for approximately $10 \%$ of the total spectrum. The electronic self-energies derived from such an excitation spectrum, using the standard momentum-independent Eliashberg formula, are shown on the same scale with experimentally derived self-energies in Figs. 4(b) and 4(c). Note that the $\operatorname{Im} \Sigma$ shown in Fig. 4(c) saturates at the higher energies as $\operatorname{Re} \Sigma$ passes through its peak. This is in sharp contrast with previously reported behavior, where $\operatorname{Re} \Sigma$ peaked at $\sim 50-70 \mathrm{meV}$ while $\operatorname{Im} \Sigma$ had a linear dependence up to the highest measured energies, clearly violating the Kramers-Kronig (KK) causality relations. While the calculated self-energies in Fig. 4 satisfy the

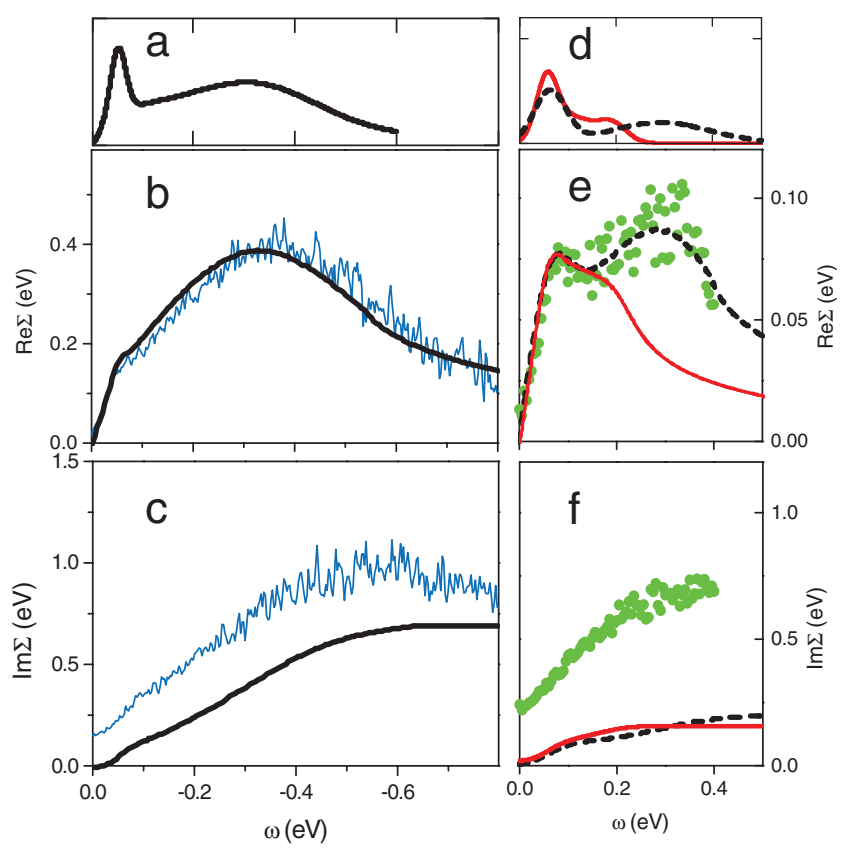

FIG. 4 (color). (a) A model for the excitation spectrum that produces self-energies similar to the measured ones in BSCCO, (b) $\operatorname{Re} \Sigma$ measured in $\mathrm{Bi} 2212$ (thin lines) and model $\operatorname{Re} \Sigma$ obtained from the spectrum in (a) (bold line). (c) Corresponding $\operatorname{Im} \Sigma$. (d)-(f) Same for LBCO at $x=1 / 8$. Solid lines represent neutron scattering data from [17] and the self-energies derived from them. Dashed lines represent the excitation spectrum and derived self-energies that better model the high-energy region of measured self-energies (green circles). 
KK relations exactly, we note that the measured ones deviate only slightly (in the BSCCO case), suggesting that the TB bands represent good approximations to the bare dispersions and that the self-energies derived here have physical meaning.

Recently, neutron scattering experiments have been performed on $\mathrm{La}_{2-x} \mathrm{Ba}_{x} \mathrm{CuO}_{4}$ [17], and on underdoped YBCO $[18,19]$, up to high energies $(\sim 300 \mathrm{meV})$, showing that the spin excitations are remarkably similar among different families of cuprate superconductors, consisting of a commensurate $(\pi, \pi)$ scattering at some finite energy $\Omega_{\text {res }}$, and scattering branches dispersing downward and upward out of this $(\pi, \pi)$ mode with increasing incommensurability. These experiments provide a unique opportunity to directly compare spin-fluctuation spectra with the electronic selfenergies within the full energy range relevant for spin dynamics for the first time. In Fig. 4(e) and 4(f) we compare the measured self-energies for LBCO at $x=$ $1 / 8$ with those modeled by using the $\mathrm{La}_{2-x} \mathrm{Ba}_{x} \mathrm{CuO}_{4}$ susceptibilities from Ref. [17]. The latter is shown in Fig. 4(d) as a solid line. We also show in Fig. 4(d) a modification to the excitation spectrum that better reproduces the measured self-energies at high energies (dashed lines). We note that, similar to BSCCO and CCOC, additional weight is always needed at energies that go somewhat beyond a typical "single-magnon" (spin-fluctuation) continuum $\sim 2-3 \mathrm{~J}$, suggesting that scattering on the two-magnon continuum might well make a contribution.

As for the BSCCO self-energies near optimal doping, we note that the low-energy $(\leq 50 \mathrm{meV})$ spin susceptibility in these systems is markedly temperature dependent. In the superconducting state, the spin spectrum is gapped and relatively well-defined modes and a strong commensurate "resonance" exist, while in the normal state, or above the pseudogap temperature, in the underdoped systems [20], the spin gap closes, and the excitations get overdamped and lose identity. Because of these changes, the quasiparticle self-energies at low energies are also temperature dependent as shown in Fig. 3(a) for optimally doped BSCCO and in general agreement with previous experiments [6,21,22].

The overall evidence shows that the high-energy kink seen in photoemission most likely results from the highenergy spin excitations. Phonons do not exist at this energy scale, and magnetic interactions are very strong in this material. The relevant high-energy spin excitations are the short wavelength nearest-neighbor spin-flip transitions. Experimentally it has been shown that in the undoped material such high-energy excitations are characterized by the $q$ vectors on the magnetic Brillouin zone boundary, i.e., the $( \pm \pi, 0)$ to $(0, \pm \pi)$ lines [16]. The coupling of quasiparticles to spin excitations with these energies and momenta is shown [23-25] to be proportional to $\cos \left(k_{x}-\right.$ $\left.q_{x}\right)+\cos \left(k_{y}-q_{y}\right)$. The nodal line will therefore couple preferentially to $q=(\pi / 2, \pi / 2)$ and $q=(-\pi / 2,-\pi / 2)$, while on moving away from the nodal direction, coupling to $q=( \pm \pi, 0)$ and $q=(0, \pm \pi)$ excitations becomes pre- dominant. However, as the hole at $\sim 300 \mathrm{meV}$ must end up at the Fermi surface, the scattering into the antinodal regions $[k \approx( \pm \pi, 0)$ and $k \approx(0, \pm \pi)]$ will always be dominant and will become stronger on moving away from the nodal line as is indeed observed here in LBCO [Fig. 3(b)] and in Ref. [11] in BSCCO.

The data also reinforce the possibility that the lowenergy kink in the superconducting state reflects the formation of the resonant mode within the spin-fluctuation spectrum. If the high-energy spin fluctuations, related to the nearest-neighbor spin-flip transitions, are able to produce the high-energy renormalization, then it is evident that the small structures at the low-energy side of the spinfluctuation spectrum, that develop upon doping and have a pronounced temperature dependence, can produce the lowenergy kink. Then, in agreement with previous studies $[6,21,22]$, only the low-energy part of the single-particle self-energy shows a significant temperature and doping dependence [Fig. 3(a)].

We acknowledge useful discussions with Andrey Chubukov, Mike Norman, Maurice Rice, and John Tranquada. The research work described in this Letter was supported by the Office of Science, U.S. Department of Energy.

*Email address: valla@bnl.gov

[1] T. Valla, A. V. Fedorov, P. D. Johnson, and S. L. Hulbert, Phys. Rev. Lett. 83, 2085 (1999).

[2] T. Valla et al., Science 285, 2110 (1999).

[3] P. V. Bogdanov et al., Phys. Rev. Lett. 85, 2581 (2000).

[4] A. Kaminski et al., Phys. Rev. Lett. 86, 1070 (2001).

[5] A. Lanzara et al., Nature (London) 412, 510 (2001).

[6] P. D. Johnson et al., Phys. Rev. Lett. 87, 177007 (2001).

[7] X. J. Zhou et al., Nature (London) 423, 398 (2003).

[8] A. D. Gromko et al., Phys. Rev. B 68, 174520 (2003).

[9] T. K. Kim et al., Phys. Rev. Lett. 91, 167002 (2003).

[10] J. Graf et al., cond-mat/0607319.

[11] B. P. Xie et al., cond-mat/0607450.

[12] R.S. Markiewicz et al., Phys. Rev. B 72, 054519 (2005).

[13] F. Ronning et al., Phys. Rev. B 71, 094518 (2005).

[14] A. A. Kordyuk et al., Phys. Rev. B 71, 214513 (2005).

[15] T. Valla, A. V. Fedorov, Jinho Li, J. S. Davis, and G. D. Gu, Science 314, 1914 (2006).

[16] R. Coldea et al., Phys. Rev. Lett. 86, 5377 (2001).

[17] J. M. Tranquada et al., Nature (London) 429, 534 (2004).

[18] S. M. Hayden, Nature (London) 429, 531 (2004).

[19] C. Stock et al., Phys. Rev. B 71, 024522 (2005).

[20] P. Dai et al., Science 284, 1344 (1999).

[21] T. Valla et al., Phys. Rev. B 73, 184518 (2006).

[22] T. Yamasaki et al., cond-mat/0603006.

[23] S. Schmitt-Rink, C. M. Varma, and A. Ruckenstein, Phys. Rev. Lett. 60, 2793 (1988).

[24] C. L. Kane, P. A. Lee, and N. Read, Phys. Rev. B 39, 6880 (1989).

[25] W.-G. Yin, C.-D. Gong, and P. W. Leung, Phys. Rev. Lett. 81, 2534 (1998). 